\title{
AN ASYMPTOTIC FORMULA FOR $a$-TH POWERS DIVIDING BINOMIAL COEFFICIENTS
}

\section{J. W. SANDER}

§1. Introduction. In 1985, Sárkôzy [11] proved a conjecture of Erdős [2] by showing that the greatest square factor $s(n)^{2}$ of the "middle" binomial coefficient $\left(\begin{array}{c}2 n \\ n\end{array}\right)$ satisfies for arbitrary $\varepsilon>0$ and sufficiently large $n$

$$
e^{(C-\varepsilon) \sqrt{n}}<s(n)<e^{(C+\varepsilon) \sqrt{n}},
$$

where

$$
C=\frac{\sqrt{2}}{2} \sum_{k=1}^{\infty}\left(\frac{1}{\sqrt{2 k-1}}-\frac{1}{\sqrt{2 k}}\right)=\left(\frac{\sqrt{2}}{2}-1\right) \zeta\left(\frac{1}{2}\right) .
$$

In the following years, several results related to prime square factors of binomial and multinomial coefficients were obtained (see [6]-[9]).

Erdôs's stronger conjecture concerning $a$-th powers dividing binomial coefficients was proved by the author [10] who showed that, for any $a \geqslant 2,0<\varepsilon<1$ and $0 \leqslant k \leqslant m$ satisfying

$$
|m-2 k|<m^{1-\varepsilon},
$$

there is always an arbitrarily large prime $p$ such that

$$
p^{a} \mid\left(\begin{array}{l}
m \\
k
\end{array}\right)
$$

if $m$ is sufficiently large. For references to problems and results concerning divisors of binomial coefficients, the reader may consult [3] or [4].

In this paper, we will generalize Sárkózy's theorem to $a$-th powers dividing binomial coefficients $\left(\begin{array}{c}2 n+a \\ n\end{array}\right)$ for "comparatively small" $d$. For this reason, we define for $a \geqslant 2$ and $|d| \leqslant n$ the integer $s_{a}(n, d)$ by

$$
\left(\begin{array}{c}
2 n+d \\
n
\end{array}\right)=s_{a}(n, d)^{a} q_{a}(n, d)
$$

with $q_{a}(n, d)$ not being divisible by an $a$-th prime power. Constants $c_{1}, c_{2}, c_{3}$ as well as implicit constants may only depend on the parameter $a$.

THEOREM 1. Let $a \geqslant 2$ and $0<\varepsilon \leqslant 1$. If

$$
|d| \ll \frac{n^{1 / a}}{(\log n)^{1+\varepsilon}},
$$

then we have for sufficiently large $n$

$$
\log s_{a}(n, d)=C_{a} n^{1 / a}+O\left(\frac{n^{1 / a}}{(\log n)^{\varepsilon}}\right),
$$


where

$$
C_{a}=2^{1 / a}\left(\frac{1}{2}\right)^{a-1} \sum_{k=1}^{\infty}\left(\left(\frac{1}{2 k-1}\right)^{1 / a}-\left(\frac{1}{2 k}\right)^{1 / a}\right)=\left(2^{1 / a}-2\right)\left(\frac{1}{2}\right)^{a-1} \zeta\left(\frac{1}{a}\right) .
$$

For $a=2$ and $d=0$, Theorem 1 implies Sárkőzy's result. For a wider range of $d$, we can show the following.

TheOrem 2. Let $a \geqslant 2$ and $0<\varepsilon<1$. If

$$
|d|<n^{1-\varepsilon},
$$

then we have for any $\varepsilon^{\prime}>0$ and sufficiently large $n$

$$
n^{\varepsilon /\left(a+1+\varepsilon+e^{\prime}\right)}<\log s_{a}(n, d)<n^{1 / a} .
$$
all

Remark. The proof of Theorem 2 shows that the upper bound holds for

$$
|d|<\frac{n^{b / a}}{(\log n)^{b+\varepsilon}},
$$

where $1 \leqslant b \leqslant a$. In this case, we have

$$
\begin{aligned}
\log s_{a}(n, d) \leqslant & 2^{1 / a}\left(\frac{1}{2}\right)^{a-b} \sum_{k=1}^{\infty}\left(\left(\frac{1}{2 k-1}\right)^{1 / a}-\left(\frac{1}{2 k}\right)^{1 / a}\right) n^{1 / a} \\
& +O\left(\frac{n^{1 / a}}{(\log n)^{\varepsilon}}\right) .
\end{aligned}
$$

The proof also implies an explicit constant for the lower bound in (3), namely

$$
\log s_{a}(n, d) \geqslant\left(\frac{1}{3}\right)^{a+1}\left(2^{\varepsilon /\left(a+1+\varepsilon+\varepsilon^{\prime}\right)}-1\right) n^{\varepsilon /\left(a+1+\varepsilon+\varepsilon^{\prime}\right)} .
$$

\$2. An asymptotic formula concerning fractional parts. As an application of a new exponential sum estimate, we recently proved

LemMA 1 ([10], Theorem 3$)$. Let $J \geqslant 1,2 \leqslant P \leqslant n^{1 / J}, \sigma=\left(\sigma_{1}, \ldots, \sigma_{J}\right)$ with $0<\sigma_{j} \leqslant 1$ for $1 \leqslant j \leqslant J$ and

$$
D(\boldsymbol{\sigma})=D(\boldsymbol{\sigma} ; P, n)=\operatorname{card}\left\{p \leqslant P:\left\{\frac{n}{p^{j}}\right\}<\sigma_{j} \quad(1 \leqslant j \leqslant J)\right\},
$$

where $\{x\}=x-[x]$ denotes the fractional part of the real number $x$. Then, for arbitrary $\varepsilon>0$, there is a positive constant $c_{1}$ such that

$$
D(\boldsymbol{\sigma})=\sigma_{1} \ldots \sigma_{J} \pi(P)+O\left(\left(P^{1-c_{1}(\log P / \log n)^{2}}+P^{(J+2) / 2+\varepsilon} n^{-1 / 2}\right)(\log n)^{4 J}\right) .
$$

COROLlary. Let $a \geqslant 2$ and $0 \leqslant \rho_{j}<\tau_{j} \leqslant 1$ for $1 \leqslant j<a$. If

$$
n^{1 / a^{2}} \leqslant P<n^{1 /(a-1)-1 / a^{2}},
$$


then

$$
\sum_{\substack{p \leqslant P \\ \rho_{j} \leqslant\left\{n / p^{j}\right\}<\tau_{j}}} \log p=\prod_{j=1 \leqslant j<a)}^{a-1}\left(\tau_{j}-\rho_{j}\right) P+O(P \exp (-\sqrt{\log P})) .
$$

Proof. We have

$$
\sum_{\substack{p \leqslant P \\ \rho_{j} \leqslant\left\{n / p^{j}\right\}<\tau_{j}}} \quad 1=\sum_{j=0}^{a-1}(-1)^{j} \sum_{\substack{i \leq\{1, \ldots, a-1\} \\|i|=j}} D_{i}(\rho, \tau),
$$

where

$$
D_{i}(\boldsymbol{\rho}, \boldsymbol{\tau})=D(\boldsymbol{\sigma})
$$

with

$$
\sigma_{j}= \begin{cases}\rho_{j}, & \text { for } j \in i, \\ \tau_{j}, & \text { for } j \notin i,\end{cases}
$$

for $0 \leqslant j<a$. By Lemma 1 , we get with $J=a-1$

$$
\begin{aligned}
& \sum_{\substack{p \leqslant P \\
\rho_{j} \leqslant\left\{n / p^{j}\right\} \tau_{j}}} 1=\prod_{j=1}^{a-1}\left(\tau_{j}-\rho_{j}\right) \pi(P) \\
&+O\left(\left(P^{1-c_{1} / a^{2}}+P^{(a+1) / 2-\left(a^{2}(a-1) / 2\left(a^{2}-a+1\right)\right)}\right)(\log n)^{4 a}\right) \\
&= \prod_{j=1}^{a-1}\left(\tau_{j}-\rho_{j}\right) \pi(P)+O\left(P^{1-c_{2}}(\log n)^{4 a}\right)
\end{aligned}
$$

for suitable $\varepsilon$ and some $c_{2}>0$. Notice that we did not make use of the lower bound in (7) up to this point.

By partial summation, we thus deduce from (8) and the prime number theorem in terms of $\theta(x)=\sum_{p \leqslant x} \log p$ with a sufficiently good error term (see for instance [1], p. 113)

$$
\begin{aligned}
& \sum_{\substack{p \leqslant P \\
\rho_{j} \leqslant\left\{n / p^{j}\right\}<\tau_{j}}} \log p=\log P \sum_{\substack{p \leqslant P \\
(1 \leqslant a)}} 1 \\
& -\int_{2}^{P}\left(\sum_{\substack{p \leqslant t \\
\rho_{j} \leqslant\left\{n / p^{j}\right\}<\tau_{j}}} 1\right) \frac{d t}{t} \\
& =\prod_{j=1}^{a-1}\left(\tau_{j}-\rho_{j}\right)\left(\pi(P) \log P-\int_{2}^{P} \frac{\pi(t)}{t} d t\right) \\
& +O\left(P^{1-c_{2}}(\log n)^{4 a} \log P\right) \\
& =\prod_{j=1}^{a-1}\left(\tau_{j}-\rho_{j}\right) \theta(P)+O\left(P^{1-c_{2}}(\log n)^{4 a} \log P\right) \\
& =\prod_{j=1}^{a-1}\left(\tau_{j}-\rho_{j}\right) P+O(P \exp (-\sqrt{\log P})) \\
& +O\left(P^{1-c_{2}}(\log n)^{4 a} \log P\right) .
\end{aligned}
$$

By the lower bound in (7), the corollary follows. 
§3. Proof of Theorem 1. First we show that, without loss of generality, $d \geqslant 0$. Suppose $d<0$. Since

$$
\left(\begin{array}{c}
2 n+d \\
n
\end{array}\right)=\left(\begin{array}{c}
2 n_{1}+d_{1} \\
n_{1}
\end{array}\right)
$$

for $n_{1}=n+d$ and $d_{1}=-d>0$, it suffices to prove that

$$
d_{1}<\frac{n_{1}^{1 / a}}{\left(\log n_{1}\right)^{1+\varepsilon}},
$$

because then, by the theorem,

$$
\begin{aligned}
\log s_{a}(n, d) & =\log s_{a}\left(n_{1}, d_{1}\right)=C_{a} n_{1}^{1 / a}+O\left(\frac{n_{1}^{1 / a}}{\left(\log n_{1}\right)^{\varepsilon}}\right) \\
& =C_{a} n^{1 / a}+O\left(\frac{n^{1 / a}}{(\log n)^{\varepsilon}}\right) .
\end{aligned}
$$

But (9) follows easily from (1). Therefore, we may assume in the sequel

$$
0 \leqslant d<\frac{n^{1 / a}}{(\log n)^{1+\varepsilon}} .
$$

Let

$$
\left(\begin{array}{c}
2 n+d \\
n
\end{array}\right)=\prod_{p \leqslant 2 n+d} p^{\beta_{p}}
$$

say. Then, for all $p$,

$$
\beta_{p}=\sum_{\alpha>0}\left(\left[\frac{2 n+d}{p^{\alpha}}\right]-\left[\frac{n}{p^{\alpha}}\right]-\left[\frac{n+d}{p^{\alpha}}\right]\right) \leqslant \sum_{\alpha=1}^{[\log (2 n+d) / \log p]} 1 \leqslant \frac{\log (2 n+d)}{\log p},
$$

thus

$$
p^{\beta_{p}} \leqslant 2 n+d .
$$

Moreover, we have for $(2 n+d)^{1 / a+1}<p \leqslant(2 n+d)^{1 / a}$

$$
\begin{aligned}
\beta_{p}=\left(\left[\frac{2 n+d}{p}\right]-\left[\frac{n}{p}\right]\right. & \left.-\left[\frac{n+d}{p}\right]\right)+\ldots \\
& +\left(\left[\frac{2 n+d}{p^{a}}\right]-\left[\frac{n}{p^{a}}\right]-\left[\frac{n+d}{p^{a}}\right]\right),
\end{aligned}
$$

and for $(2 n+d)^{1 / a}<p \leqslant 2 n+d$

$$
\beta_{p} \leqslant a-1
$$

Define

$$
\gamma_{p}=a\left[\frac{\beta_{p}}{a}\right]
$$

such that

$$
s_{a}(n, d)^{a}=\prod_{p \leqslant 2 n+d} p^{\gamma_{p}}
$$


Obviously,

$$
\gamma_{p} \leqslant \beta_{p}
$$

and, by (13), we have for $(2 n+d)^{1 / a}<p \leqslant 2 n+d$

$$
\gamma_{p}=0 \text {. }
$$

Now, setting

$$
T=T(n)=\left[(\log n)^{a}\right]
$$

and

$$
X=X(n)=\left(\frac{2 n+d}{T+1}\right)^{1 / a},
$$

we deduce from (15) and (17) that

$$
s_{a}(n, d)^{a}=\prod_{p \leqslant(2 n+d)^{1 / a}} p^{\gamma_{p}}=U_{0} U
$$

where

$$
U_{0}=\prod_{p \leqslant X} p^{\gamma_{p}}
$$

and

$$
U=\prod_{X<p \leqslant(2 n+d)^{1 / a}} p^{\gamma_{p}} .
$$

Collecting (11), (16) and (10), we obtain by Chebyshev's theorem ([1], p. 55) for sufficiently large $n$

$$
\begin{aligned}
\log U_{0}=\sum_{p \leqslant X} \log p^{\gamma_{p}} & \leqslant \sum_{p \leqslant X} \log p^{\beta_{p}} \leqslant \sum_{p \leqslant X} \log (2 n+d) \\
& <2 \log n \frac{X}{\log X} \\
& \leqslant 2 \log n \frac{(3 n)^{1 / a}}{\log n}\left(\frac{1}{a} \log 2 n-\log \log n\right)^{-1} \\
& \leqslant 12 a \frac{n^{1 / a}}{\log n} .
\end{aligned}
$$

Now we turn our attention to $U$. By (12) and (14), we get

$$
\log U=\sum_{x<p \leqslant(2 n+d)^{1 / a}} \gamma_{p} \log p=a \sum_{\substack{x<p \leqslant(2 n+d)^{1 / a} \\ E_{1}(j)}} \log p
$$

where $E_{1}(j)$ denotes the condition

$$
\left[\frac{2 n+d}{p^{j}}\right]-\left[\frac{n}{p^{j}}\right]-\left[\frac{n+d}{p^{j}}\right]=1
$$

It is easily seen that, for real numbers $x$ and $0 \leqslant \delta<1$,

$$
[2 x+\delta]-[x]-[x+\delta]= \begin{cases}1, & \text { for } 1-\delta \leqslant 2\{x\}<2-2 \delta \text { or } 2-\delta \leqslant 2\{x\} \\ 0, & \text { for } 2\{x\}<1-\delta \text { or } 2-2 \delta \leqslant 2\{x\}<2-\delta\end{cases}
$$


By (10), we have for $1 \leqslant j<a, p>X$ and sufficiently large $n$

$$
0 \leqslant \frac{d}{p^{j}}<\frac{d}{X} \leqslant d\left(\frac{2 T}{2 n}\right)^{1 / a} \leqslant \frac{d \log n}{n^{1 / a}} \leqslant c_{3} \lambda<1
$$

with some $c_{3}>0$ and

$$
\lambda=\lambda(n)=\frac{1}{(\log n)^{\varepsilon}} .
$$

Setting

$$
x=\frac{n}{p^{j}}, \quad \delta=\frac{d}{p^{j}},
$$

we thus obtain from (20) and (21)

$$
\log U=a \sum_{E_{2}(j) \text { or } E_{3}(j)(2 n+d)^{1 / a}(1 \leqslant a), \quad E_{1}(a)} \log p,
$$

where $E_{2}(j)$ and $E_{3}(j)$ denote the conditions

$$
1-\frac{d}{p^{j}} \leqslant 2\left\{\frac{n}{p^{j}}\right\}<2-\frac{2 d}{p^{j}}
$$

and

$$
2-\frac{d}{p^{j}} \leqslant 2\left\{\frac{n}{p^{j}}\right\} .
$$

By (22), we have for $1 \leqslant j<a$

$$
\begin{aligned}
\sum_{\substack{E(p) \\
E_{2}(j) \text { or } E_{3}(j)}} \log p & =\sum_{\substack{E(p) \\
E_{2}(j)}} \log p-\sum_{\substack{E(p) \\
E_{3}(j)}} \log p \\
& =\sum_{\substack{E(p) \\
E_{2}(j)}} \log p+O\left(\sum_{\substack{X<p \leqslant(2 n+d)^{1 / a} \\
2-c_{3} \lambda \leqslant 2\left\{n / p^{\prime}\right\}}} \log p\right),
\end{aligned}
$$

where $E(p)$ indicates that $p$ is subject to some arbitrary conditions including $X<p \leqslant(2 n+d)^{1 / a}$. Applying this process successively to the sum in (23) for $j=1,2, \ldots, a-1$, we deduce

$$
\log U=a \sum_{\substack{x<p \leq(2 n+d) \\ E_{2}(j)}} \log p+O\left(\max _{\substack{1 \leqslant j<a), 1 \leqslant j<a}} \sum_{\substack{x<p \leq(2 n+d) \\ 1-c_{3} \lambda \leqslant\left\{n / p^{\prime}\right\}}} \log p\right) .
$$

Again by (22), we have for $1 \leqslant j<a$

$$
\begin{aligned}
\sum_{\substack{E(p) \\
E_{2}(j)}} \log p & =\sum_{\substack{E(p) \\
\left\{n / p^{j}\right\} \geqslant \frac{1}{2}}} \log p+\sum_{\substack{E(p) \\
1-\left(d / p^{\prime}\right) \leqslant 2\left\{n / p^{j}\right\}<1}} \log p-\sum_{\substack{E(p) \\
\left\{n / p^{j}\right\} \geqslant 1-\left(d / p^{j}\right\}}} \log p \\
& =\sum_{\substack{E(p) \\
\left\{n / p^{j}\right\} \geqslant \frac{1}{2}}} \log p+O\left(\sum_{\substack{x<p \leqslant\left\{(2 n+d)^{1 / a} \\
1-c_{3} \lambda \leqslant 2\left\{n / p^{j}\right\}<1\right.}} \log p\right)+O\left(\sum_{\substack{x<p \leqslant\left\{(2 n+d)^{1 / a} \\
\left\{n / p^{j}\right\} \geqslant 1-c_{3} \lambda\right.}} \log p\right)
\end{aligned}
$$

with $E(p)$ as above. Using this equality successively in (24) for 
$j=1,2, \ldots, a-1$, we obtain

$$
\begin{aligned}
\log U=a & \sum_{\substack{x<p \leqslant(2 n+d)^{1 / a} \\
\left\{n / p^{j}\right\} \geqslant \frac{1}{2}}} \log p \\
+ & O\left(\max _{\substack{1 \leqslant j<a), \quad E_{1}(a) \\
1 \leqslant j<a}} \max _{\substack{I \in[0,1] \\
|I| \leqslant c_{3} \lambda}} \sum_{\substack{X<p \leqslant(2 n+d))^{1 / a} \\
\left\{n / p^{j}\right\} \in I}} \log p\right),
\end{aligned}
$$

where $I$ runs over intervals and $|I|$ denotes the length of $I$.

Obviously, by (22),

$$
n^{1 / a^{2}}<X<(2 n+d)^{1 / a}<n^{1 /(a-1)-1 / a^{2}} .
$$

Therefore, we may apply the corollary with $\rho_{j}=0, \tau_{j}=1\left(j \neq j_{0}\right)$ and $\left[\rho_{j_{0}}, \tau_{j_{0}}\right]=I$ for the appropriate $j_{0}$. Then we get from (25)

$$
\log U=a \sum_{\substack{x<p \leqslant(2 n+d)^{1 / a} \\\left\{n / p^{J}\right\} \geqslant \frac{1}{2}}} \log p+O\left(\lambda n^{1 / a}\right) .
$$

For $t \in \mathbb{N}$, we set

$$
x_{t}=\left(\frac{2 n+d}{t}\right)^{1 / a} .
$$

Then we define for $1 \leqslant t \leqslant T$ intervals

$$
I_{t}=\left\{x: x_{t+1}<x \leqslant x_{t}\right\} .
$$

For a prime $p \in I_{t}(1 \leqslant t \leqslant T)$, we have

$$
x_{t+1}=\left(\frac{2 n+d}{t+1}\right)^{1 / a}<p \leqslant\left(\frac{2 n+d}{t}\right)^{1 / a}=x_{t},
$$

that is

$$
t \leqslant \frac{2 n+d}{p^{a}}<t+1
$$

or

$$
t=\left[\frac{2 n+d}{p^{a}}\right] .
$$

Hence, we have for $X<p \leqslant(2 n+d)^{1 / a}$

$$
2 \mid\left[\frac{2 n+d}{p^{a}}\right] \Leftrightarrow p \in I_{2 k}
$$

for some $1 \leqslant k \leqslant[T / 2]$, and

$$
2 \nmid\left[\frac{2 n+d}{p^{a}}\right] \Leftrightarrow p \in I_{2 k-1}
$$

for some $1 \leqslant k \leqslant\left[\frac{1}{2}(T+1)\right]$.

For real $x$ and $0 \leqslant \delta<1$, we clearly have

$$
[2 x+\delta]-[x]-[x+\delta] \in\{0,1\}
$$


and

$$
[x+\delta]-[x] \in\{0,1\}
$$

Thus

$$
[2 x+\delta]-[x]-[x+\delta]= \begin{cases}1, & \text { if } 2 \mid[2 x+\delta] \text { and }[x] \neq[x+\delta] \\ 1, & \text { if } 2 \nmid[2 x+\delta] \text { and }[x]=[x+\delta] \\ 0, & \text { otherwise. }\end{cases}
$$

Setting $x=n / p^{a}$ and $\delta=d / p^{a}$, we obtain from (22), (28) and (29) for $X<p \leqslant(2 n+d)^{1 / a}$

$\left[\frac{2 n+d}{p^{a}}\right]-\left[\frac{n}{p^{a}}\right]-\left[\frac{n+d}{p^{a}}\right]$

$$
=\left\{\begin{array}{l}
1, \text { if } p \in I_{2 k} \text { for some } 1 \leqslant k \leqslant\left[\frac{1}{2} T\right] \text { and }\left\{n / p^{a}\right\} \geqslant 1-\left(d / p^{a}\right), \\
1, \text { if } p \in I_{2 k-1} \text { for some } 1 \leqslant k \leqslant\left[\frac{1}{2}(T+1)\right] \text { and }\left\{n / p^{a}\right\}<1-\left(d / p^{a}\right), \\
0, \text { otherwise. }
\end{array}\right.
$$

Therefore, (27) implies with (22)

$$
\begin{aligned}
& \log U=a \sum_{k=1}^{[(T+1) / 2]} \sum_{\substack{p \in I_{2 k-1},\left\{n / p^{a}\right\}<1-\left(d / p^{a}\right) \\
\left\{n / p^{\prime}\right\} \geq \frac{1}{(1 \leqslant j<a)}}} \log p \\
& +a \sum_{k=1}^{[T / 2]} \sum_{\substack{p \in I_{2 k},\left\{n / p^{a}\right\} \geqslant 1-\left(d / p^{a}\right) \\
\left\{n / p^{j}\right\} \geqslant \frac{1}{2}(1 \leqslant j<a)}} \log p+O\left(\lambda n^{1 / a}\right) \\
& =a \cdot \sum_{k=1}^{[(T+1) / 2]} \sum_{\substack{p \in I_{2 k-1} \\
\left\{n / p^{j}\right\} \geqslant \frac{1}{2}(1 \leqslant j<a)}} \log p+O\left(\sum_{\substack{x<p \leqslant(2 n+d)^{1 / a} \\
\left\{n / p^{a}\right\} \geqslant 1-\left(d / p^{a}\right)}} \log p\right) \\
& +O\left(\lambda n^{1 / a}\right) \text {. }
\end{aligned}
$$

Clearly,

$$
\left\{\frac{n}{p^{a}}\right\} \geqslant 1-\frac{d}{p^{a}} \Leftrightarrow p^{a} \mid\left(n+d_{0}\right)
$$

for some $1 \leqslant d_{0} \leqslant d$. Assume that, for fixed $d_{0}, 1 \leqslant d_{0} \leqslant d$, there is a prime $p, X<p \leqslant(2 n+d)^{1 / a}$, satisfying $p^{a} \mid\left(n+d_{0}\right)$. By the definition of $X$, we have

$$
\frac{n+d_{0}}{p^{a}}<\frac{2 n+d}{p^{a}}<(\log n)^{a}<X
$$

for sufficiently large $n$. Hence, for each $1 \leqslant d_{0} \leqslant d$, there is at most one prime $p>X$ satisfying $p^{a} \mid\left(n+d_{0}\right)$. Thus, by (31) and (10),

$$
\sum_{\substack{x<p \leqslant(2 n+d)^{1 / a} \\\left\{n / p^{3}\right\} \geqslant 1-\left(d / p^{a}\right)}} \log p=\sum_{d_{0}=1}^{d} \sum_{\substack{x<p<(2 n+d)^{1 / a} \\ p^{a} \mid\left(n+d_{0}\right)}} \log p<d \log n<\lambda n^{1 / a} .
$$


Now (30) yields

$$
\log U=a \sum_{k=1}^{\left[\frac{1}{2}(T+1)\right]} \sum_{\substack{p \in I_{2 k-1} \\\left\{n / p^{j}\right\} \geqslant \frac{1}{2}(1 \leqslant j<a)}} \log p+O\left(\lambda n^{1 / a}\right) .
$$

With regard to (26), we are able to apply the corollary to the main term in (32), namely

$$
\begin{aligned}
\sum_{\substack{p \in I_{2 k-1} \\
\left\{n / p^{j}\right\} \geqslant \frac{1}{2}(1 \leq j<a)}} \log p & =\left(\frac{1}{2}\right)^{a-1}\left(x_{2 k-1}-x_{2 k}\right)+O\left(x_{2 k-1} \exp \left(-\sqrt{\log x_{2 k-1}}\right)\right) \\
& =\left(\frac{1}{2}\right)^{a-1} D_{k}(2 n+d)^{1 / a}+O\left(\frac{n^{1 / a}}{(\log n)^{a+1}}\right)
\end{aligned}
$$

for sufficiently large $n$ and $1 \leqslant k \leqslant\left[\frac{1}{2}(T+1)\right]$, where

$$
D_{k}=\left(\frac{1}{2 k-1}\right)^{1 / a}-\left(\frac{1}{2 k}\right)^{1 / a} \text {. }
$$

Therefore, we obtain from (32)

$$
\log U=a\left(\frac{1}{2}\right)^{a-1}(2 n+d)^{1 / a} \sum_{k=1}^{\left[\frac{1}{2}(T+1)\right]} D_{k}+O\left(\frac{n^{1 / a}}{(\log n)^{a+1}} T\right)+O\left(\lambda n^{1 / a}\right) .
$$

Obviously,

$$
D_{k}=\left(\frac{1}{2 k-1}\right)^{1 / a}\left(1-\left(1-\frac{1}{2 k}\right)^{1 / a}\right) \leqslant\left(\frac{1}{2 k-1}\right)^{1 / a} \frac{1}{2 a k}<\left(\frac{1}{2 k-1}\right)^{1+(1 / a)},
$$

hence

$$
\sum_{k>\left[\frac{1}{2}(T+1)\right]} D_{k}<\left(\frac{1}{T}\right)^{1 / a} \ll \frac{1}{\log n} .
$$

Now (33) together with (22) implies

$$
\log U=a\left(\frac{1}{2}\right)^{a-1}(2 n)^{1 / a} \sum_{k=1}^{\infty} D_{k}+O\left(\lambda n^{1 / a}\right) .
$$

With (18) and (19), this completes the proof of Theorem 1.

§4. Proof of Theorem 2. We need the following old result due to Kummer, where $e(n ; p)$ denotes the order of the prime $p$ in the positive integer $n$.

LEMMA 2 ([5], p. 116). For non-negative integers $m$ and $n$,

$$
e\left(\left(\begin{array}{c}
m+n \\
m
\end{array}\right) ; p\right)
$$

equals the number of "carries" that occur when $m$ and $n$ are added in p-adic notation. 
The upper bound in (3) follows immediately for all $d \leqslant n$ from (18), (16), (12) and Chebyshev's theorem (see [1], p. 55). In order to show (5), we copy the proof of Theorem 1 with a few modifications. With (4), (22) will be replaced by

$$
0 \leqslant \frac{d}{p^{j}}<\frac{d}{X^{b}} \leqslant d\left(\frac{2 T}{2 n}\right)^{b / a} \leqslant \frac{d(\log n)^{b}}{n^{b / a}} \leqslant c_{3} \lambda<1
$$

for $b \leqslant j<a$ and $p>X$. Changing the condition $1 \leqslant j<a$ into $b \leqslant j<a$ in (24), (25) and (27), we get upper bounds for $\log U$. This finally leads to the claimed inequality (5).

In order to prove the lower bound in (3), we consider the set

$$
M=\left\{n^{1 /\left(J+\varepsilon^{\prime}\right)}<p<(2 n)^{1 /\left(J+\varepsilon^{\prime}\right)}: \frac{2}{3}<\left\{\frac{n}{p^{j}}\right\} \quad(K<j \leqslant J), \quad\left\{\frac{n}{p^{K}}\right\}<\frac{1}{2}\right\},
$$

where

$$
J=\left[\frac{a+1+\varepsilon^{\prime}}{\varepsilon}\right],
$$

and $K=J-a$. By Lemma 1 and the prime number theorem, we get

$$
\begin{aligned}
\operatorname{card} M & =\frac{1}{2}\left(\frac{1}{3}\right)^{J-K}\left(\pi\left((2 n)^{1 /\left(J+\varepsilon^{\prime}\right)}\right)-\pi\left(n^{1 /\left(J+\varepsilon^{\prime}\right)}\right)\right)+O\left(\frac{n^{1 /\left(J+\varepsilon^{\prime}\right)}}{(\log n)^{2}}\right) \\
& =C_{1} \frac{n^{1 /\left(J+\varepsilon^{\prime}\right)}}{\log n}+O\left(\frac{n^{1 /\left(J+\varepsilon^{\prime}\right)}}{(\log n)^{2}}\right)
\end{aligned}
$$

with

$$
C_{1}=\frac{1}{2}\left(\frac{1}{3}\right)^{a}\left(J+\varepsilon^{\prime}\right)\left(2^{1 /\left(U+\varepsilon^{\prime}\right)}-1\right) .
$$

For any $p \in M$, we have

$$
\begin{gathered}
\frac{1}{2} p^{J+\varepsilon^{\prime}}<n<p^{J+\varepsilon^{\prime}} \\
\left\{\frac{n}{p^{j}}\right\}>\frac{2}{3} \quad(K<j \leqslant J),
\end{gathered}
$$

and

$$
\left\{\frac{n}{p^{K}}\right\}<\frac{1}{2}
$$

Write $n$ in $p$-adic notation, namely

$$
n=n_{J} p^{J}+n_{J-1} p^{J-1}+\ldots+n_{1} p+n_{0} \quad\left(0 \leqslant n_{j}<p\right) .
$$

For $K<j \leqslant J$, we have by (36)

$$
\frac{2}{3}<\left\{\frac{n}{p^{j}}\right\}=\frac{n_{j-1} p^{j-1}+\ldots+n_{0}}{p^{j}}
$$


thus

$$
\frac{n_{j-1}}{p}>\frac{2}{3}-(p-1)\left(\frac{1}{p^{2}}+\ldots+\frac{1}{p^{j}}\right)>\frac{2}{3}-\frac{1}{p} .
$$

This implies for $p \geqslant 7$

$$
n_{j-1}>\frac{1}{2} p
$$

i.e.,

$$
n_{j}>\frac{1}{2} p \quad(K \leqslant j<J) .
$$

By (37), we get in a similar fashion

$$
n_{K-1}<\frac{1}{2} p .
$$

By (2) and (35),

$$
0 \leqslant d<n^{1-\varepsilon}<p^{\left(J+\varepsilon^{\prime}\right)\left(1-\varepsilon^{\prime}\right)}<p^{K-1},
$$

where again $d \geqslant 0$ is assumed without loss of generality. Writing $d$ in $p$-adic notation, too, we therefore get

$$
d=d_{K-2} p^{K-2}+\ldots+d_{0}
$$

with integers $d_{j}, 0 \leqslant d_{j}<p$. Thus we have by (35), (38) and (39)

$$
\begin{aligned}
n & =n_{J} p^{J}+\ldots+n_{K-1} p^{K-1}+\ldots+n_{0}, \\
n+d & =n_{J}^{\prime} p^{J}+\ldots+n_{K-1}^{\prime} p^{K-1}+\ldots+n_{0}^{\prime},
\end{aligned}
$$

where

$$
n_{j}=n_{j}^{\prime}>\frac{1}{2} p \quad(K \leqslant j<J)
$$

By Lemma 2,

$$
e\left(\left(\begin{array}{c}
2 n+d \\
n
\end{array}\right) ; p\right) \geqslant J-K=a,
$$

which means that each $p \in M$ satisfies $p^{a} \mid\left(\begin{array}{c}2 n+d \\ n\end{array}\right)$. Hence we conclude by (34)

$$
s_{a}(n, d) \geqslant \prod_{p \in M} p \geqslant n^{1 /\left(J+\varepsilon^{\prime}\right) C_{1} n^{1 /\left(J+\varepsilon^{\prime}\right) / \log n},}
$$

thus

$$
\log s_{a}(n, d) \geqslant\left(\frac{1}{3}\right)^{a+1}\left(2^{1 /\left(J+\varepsilon^{\prime}\right)}-1\right) n^{1 /\left(J+\varepsilon^{\prime}\right)}
$$

for sufficiently large $n$. By the definition of $J$, this yields the lower bound of Theorem 2 and (6).

\section{References}

1. H. Davenport. Multiplicative Number Theory, 2nd edition, revised by Hugh L. Montgomery (Springer-Verlag, New York-Heidelberg-Berlin, 1980).

2. P. Erdôs. Problems and Results on Number Theoretic Properties of Consecutive Integers and Related Questions. Proc. Fifth Manitoba Conf. on Numerical Mathematics (1975), 25 44.

3. P. Erdốs and R. L. Graham. Old and New Problems and Results in Combinatorial Number Theory. L'Enseign. Math., Geneva, 1980. 
4. R. K. Guy. Unsolved Problems in Number Theory (Springer-Verlag, New York-HeidelbergBerlin, 1981).

5. E. E. Kummer. Über die Ergänzungssätze zu den allgemeinen Reciprocitäts gesetzen. J. reine angew. Math., 44 (1852), 93-146.

6. J. W. Sander. On prime powers dividing $\left(\begin{array}{c}2 n \\ n\end{array}\right)$. To appear.

7. J. W. Sander. Prime power divisors of $\left(\begin{array}{c}2 n \\ n\end{array}\right)$, J. Number Theory, 39 (1991), 65-74.

8. J. W. Sander. On prime divisors of binomial coefficients. To appear in Bull. London Math. Soc.

9. J. W. Sander. Prime power divisors of multinomial coefficients and Artin's conjecture. To appear.

10. J. W. Sander. Prime power divisors of binomial coefficients. To appear in J. reine angew. Math. 11. A. Sárközy. On divisors of binomial coefficients, I. J. Number Theory, 20 (1985), 70-80.

Herr J. W. Sander,

Institut für Mathematik,

Universität Hannover,

Welfengarten 1 ,

3000 Hannover 1 ,

Germany.
11B65: NUMBER THEORY; Sequences and sets; Binomial coefficients.

Received on the 18th of June, 1991. 